\title{
Real-space multiple scattering method for angle-resolved photoemission and valence-band photoelectron diffraction and its application to $\mathrm{Cu}(111)$
}

\author{
P. Krüger, ${ }^{1, *}$ F. Da Pieve, ${ }^{2}$ and J. Osterwalder ${ }^{3}$ \\ ${ }^{1}$ ICB, UMR 5209 CNRS, Université de Bourgogne, F-21078 Dijon, France \\ ${ }^{2}$ EMAT, University of Antwerp, Groenenborgerlaan 171, B-2020 Antwerp, Belgium \\ ${ }^{3}$ Physik-Institut, Universität Zürich, Winterthurerstr. 190, CH-8057 Zürich, Switzerland
}

(Received 26 October 2010; revised manuscript received 18 January 2011; published 21 March 2011)

\begin{abstract}
A computational method is presented for angle-resolved photoemission spectra (ARPES) and photoelectron diffraction (PED) in the ultraviolet regime. The one-step model is employed and both initial valence and final continuum states are calculated using the finite-cluster, real-space multiple scattering method. Thereby the approach is versatile and provides a natural link to core-level PED. The method is applied to the $\mathrm{Cu}(111)$ valence band and good agreement with experiment is found for both ARPES spectra and PED patterns. When the PED patterns are integrated over a filled band of a single-orbital symmetry, such as $\mathrm{Cu}-3 d$, we show, both numerically and analytically, that the exact theory with delocalized initial states can be replaced by the much simpler, core-level-type theory where the initial states are taken as localized.
\end{abstract}

DOI: 10.1103/PhysRevB.83.115437

PACS number(s): 79.60.Bm, 73.20.At, 71.20.Gj

\section{INTRODUCTION}

Energy- and angle-resolved x-ray and ultraviolet photoemission spectroscopy are, respectively, successful techniques for complete structural characterization and for mapping electronic energy bands of solids. ${ }^{1}$ The attraction of photoelectron diffraction in the $\mathrm{x}$-ray regime lies in the possibility of obtaining information on the structural properties simultaneously with chemical selectivity. Experimental patterns can be accurately reproduced within the multiple scattering approach $^{2}$ and complete angular intensity mappings can be used to form electron holograms. ${ }^{3,4}$ In contrast, the ultraviolet regime allows study of photoemission from electronic states close to the Fermi level, which are responsible for the chemical and the low-energy physical properties of the system and thus determine many interesting phenomena, such as metal-insulator transitions and high- $T_{c}$ superconductivity. Angle-resolved photoemission spectroscopy (ARPES) in the ultraviolet region is nowadays the most used technique for momentum space microscopy. ${ }^{5}$ Recently, spin-resolved ARPES has also become an interesting tool to study the spin-polarized Fermi surface of magnetic materials. ${ }^{6}$ The data analysis of ultraviolet valence band photoemission spectra relies on the conservation laws of energy and momentum in the photoemission process. In the ultraviolet regime the photon momentum is negligible in the photoemission process. ${ }^{7}$ Thus the spectra show peaks at those photoelectron energies and momenta where $k$-vector conserving transitions exist between the occupied and the unoccupied bands. Collecting the photoelectron emission intensities over the valence band region for different detection angles allows, in principle, ${ }^{8}$ mapping of the band structure of the system.

Diffraction patterns of low-energy electrons are not often studied in solid state physics, because in this case a clear interpretation of the experimental data is missing, due both to (i) the need for an appropriate description that combines the structural and band states properties and (ii) the multiple scattering events felt by low-kinetic-energy outgoing electrons. The electrons are emitted from states that are delocalized and form the energy bands of the sample, and thus the initial state reflects the hybridization properties of the system and can be strongly sensitive to the difference between bulk and surface emitter sites. A simple structural interpretation as in core-level photoelectron diffraction is not valid for the valence band, which makes the analysis of the angular dependence more difficult. However, several interesting experimental studies on diffraction patterns have been performed during the last decades. Osterwalder et al. ${ }^{9}$ studied valence band photoelectron diffraction from $\mathrm{Cu}(111)$ in the ultraviolet (UV) regime. The energy-integrated diffraction patterns could be successfully modeled using single scattering calculations with localized initial states. Stuck et al. ${ }^{10}$ performed valence band diffraction patterns with high-kinetic-energy photoelectrons to extract the chemically resolved partial density of states (DOS) of alloys. Sondergaard et al. ${ }^{11}$ used such angular patterns to break down the DOS into different $l$-resolved contributions. Angle-resolved photoemission is also used for free clusters, which are interesting systems both for a fundamental understanding of the many-body problem and for practical nanotechnological applications. ${ }^{12,13}$ The study of the angular distribution of photoelectrons can unravel cluster size effects that underline the transition from individual atoms and molecules to larger systems, finally evolving to the solid state behavior. ${ }^{14}$

The multiple scattering method has proven to be a powerful and flexible approach for various electron spectroscopies, including $\mathrm{x}$-ray photoemission and $\mathrm{x}$-ray photoelectron diffraction (XPD). ${ }^{2}$ The real-space multiple scattering (RSMS) formulation was tailored to core excitations in the $\mathrm{X}$-ray regime and there is no counterpart for photoemission in the low-energy, that is, UV regime. In existing RSMS implementations, recent effort has been devoted to an $a b$ initio description of various spectroscopies over a wide energy range, ${ }^{15}$ but ARPES and photoelectron diffraction in the UV regime are not included in these developments. The RSMS approach relies on the use of finite-size clusters, in contrast to band structure codes that model the system as an infinite crystal. 
In this paper we present the development of a RSMS method for valence band photoemission and its application to ARPES and valence band photoelectron diffraction (PED) at the $\mathrm{Cu}(111)$ surface excited with UV light. The calculations are compared with available experimental data, namely energy spectra at various emission angles (ARPES) and full PED patterns for finite energy intervals in the valence band. We find good agreement both for ARPES and PED. In the case of ARPES the agreement is comparable with that of $k$-space methods, which shows that $k_{\|}$conservation does not need to be implemented explicitly to obtain reliable results. In the PED part, we make extensive comparison with the data by Osterwalder et al. ${ }^{9,16}$. We show both numerically and analytically that for PED patterns integrated over the whole Cu-3d band, the exact theory with delocalized initial states can be replaced by a simplified XPD-type theory with localized initial states. This was already suggested by Osterwalder et $^{\mathrm{al}}{ }^{9}$ and the present results provide a proof of this conjecture.

The paper is organized as follows: in Sec. II we present the theoretical approach, and in Secs. III and IV we study, respectively, ARPES and PED calculations on $\mathrm{Cu}(111)$ and compare the results with experimental data. In Sec. V we draw conclusions.

\section{THEORY}

The photoemission intensity for photons of energy $\hbar \omega$ and polarization $q$ is given by

$$
I\left(\omega, q, \mathbf{k}^{\prime}\right) \sim \sum_{\nu}\left|\left\langle\mathbf{k}^{\prime}\left|D_{q}\right| \nu\right\rangle\right|^{2} \delta\left(E_{\mathbf{k}^{\prime}}-E_{\nu}-\hbar \omega\right) .
$$

Here, $\left|\mathbf{k}^{\prime}\right\rangle$ is final state of the photoelectron detected with momentum $\mathbf{k}^{\prime}$. This photoelectron wave is described as a time-reversed LEED state, that is, as the sum of a plane wave $\exp \left(i \mathbf{k}^{\prime} \mathbf{r}\right)$ and spherical waves incoming at each atomic site. $|v\rangle$ is an initial state with energy $E_{v}$. We use the dipole approximation of the optical transition, and $D_{q}$ is the dipole operator for $q$-polarized light. Here we focus on nonmagnetic systems, so electron spin does not appear explicitly in Eq. (1). We describe both the initial and the final states in RSMS theory. For a point $\mathbf{r}$ inside the finite cluster the wave functions are given by

$$
\langle\mathbf{r} \mid \mathbf{k}\rangle=\sum_{i L} B_{i L}(\mathbf{k}) \phi_{i L}\left(\mathbf{r}, E_{k}\right),
$$

where $\phi_{i L}\left(\mathbf{r}, E_{k}\right)$ is the solution of the Schrödinger equation for the single scatterer at site $i$ with angular momentum $L \equiv(l, m)$, energy $E_{k}$, and $T$-matrix normalization. ${ }^{17}$

$B_{i L}(\mathbf{k})$ are the multiple scattering amplitudes. Here $\mathbf{k}$ denotes the wave vector of the outgoing plane wave inside the matter. It is related to the wave vector in the asymptotic region $\mathbf{k}$ ' through conservation of energy and momentum parallel to the surface. We have $\mathbf{k}_{\|}^{\prime}=\mathbf{k}_{\|}$and $\left(k_{\perp}^{\prime}\right)^{2}=k_{\perp}^{2}-V_{\text {in }}$, where $V_{\text {in }}$ is the so-called inner potential, which physically represents the height of the surface potential barrier. In muffintin approximation $V_{\text {in }}$ is given by the difference between the vacuum level and the interstitial potential $V_{0}$. We have $V_{\text {in }}=W+E_{F}-V_{0}$, where $W$ is the work function and $E_{F}$ the Fermi level. In the numerical calculations we took $W=4.5 \mathrm{eV}$ from experiment and $V_{0}$ and $E_{F}$ from a self-consistent band structure calculation of bulk $\mathrm{Cu}$.

The multiple scattering amplitudes are given by ${ }^{2}$

$$
B_{i L}(\mathbf{k})=\sqrt{\frac{k}{\pi}} \sum_{j L^{\prime}} \tau_{L L^{\prime}}^{i j} i^{l^{\prime}} Y_{L^{\prime}}(\hat{\mathbf{k}}) e^{i \mathbf{k} \cdot R_{j 0}},
$$

where $\tau_{L L^{\prime}}^{i j}$ is the scattering path operator, which represents the probability amplitude for the electron to propagate from site $i$ with angular momentum $L$ to site $j$ with angular momentum $L^{\prime}$. The scattering path operator is obtained by inversion of the matrix $\left[t^{-1}-G\right]$; see, for example, Ref. 2 for details. The other factors on the right-hand side of Eq. (3) come from the development of the outgoing plane wave in terms of spherical waves.

If we took localized orbitals as initial states $|v\rangle$ in Eq. (1), the foregoing formulas would yield the usual multiple scattering theory for core-level XPD. ${ }^{2}$ In the present case of valence band photoemission, the initial states are delocalized and we develop them in RSMS with Eq. (3). The sum over initial states in Eq. (1) can then be avoided by virtue of the optical theorem, ${ }^{2}$ which we write as

$$
\sum_{\nu}\langle\mathbf{r} \mid v\rangle\left\langle v \mid \mathbf{r}^{\prime}\right\rangle \delta\left(E-E_{v}\right)=\sum_{i j L L^{\prime}} \phi_{i L}\left(\mathbf{r}, E_{v}\right) I_{L L^{\prime}}^{i j} \phi_{j L^{\prime}}^{*}\left(\mathbf{r}^{\prime}, E_{v}\right),
$$

where

$$
I_{L L^{\prime}}^{i j} \equiv-\frac{1}{2 i \pi}\left(\tau-\tau^{\dagger}\right)_{L L^{\prime}}^{i j}
$$

is essentially the imaginary part of the scattering path operator. The photoemission cross section becomes

$$
I\left(\omega, q, \mathbf{k}^{\prime}\right) \sim \sum_{i j L L^{\prime}} M_{i L}^{q}(\mathbf{k}) I_{L L^{\prime}}^{i j}\left(E_{k}-\hbar \omega\right) M_{j L^{\prime}}^{q *}(\mathbf{k}),
$$

where

$$
M_{i L}^{q}(\mathbf{k})=\sum_{L^{\prime \prime}} B_{i L^{\prime \prime}}^{*}(\mathbf{k})\left\langle\phi_{i L^{\prime \prime}}\left(E_{k}\right)\left|D_{q}\right| \phi_{i L}\left(E_{k}-\hbar \omega\right)\right\rangle .
$$

There is no sum over sites in Eq. (6) because the dipole operator is local. The sum over $L^{\prime \prime}$ is limited to the two terms with $l^{\prime \prime}=l \pm 1$ and $m^{\prime \prime}=m+q$.

We note that the present formalism is fully equivalent to one-step ARPES theory with the layered KKR method. ${ }^{18,19}$ The only difference is that we do not rely on translational invariance in the surface plane, but we use the RSMS approach for all three dimensions. While the layered KKR approach has obvious numerical advantages for systems with small surface unit cells, the present scheme appears more flexible and its link to core-level XPD is more direct.

Contrary to core-level XPD, the waves emitted from different sites interfere coherently in valence band photoemission. This coherence is established in Eq. (5) through the imaginary part of the scattering path operator $I^{i j}$. If we make a singlesite approximation, $I^{i j} \sim \delta_{i j}$, then coherence is lost and the standard XPD formulas are recovered. In contrast, one may also apply some approximation to the scattering path operator used for the calculation of the final-state multiple scattering amplitudes $B_{i L}(\mathbf{k})$ in Eq. (3). If one puts, for example, $\tau_{L L^{\prime}}^{i j}=$ $\delta_{i j} \delta_{L L^{\prime}}$, then all final-state scattering is neglected. If one, 
moreover, replaces $\phi_{L^{\prime \prime}}\left(E_{k}\right)$ in Eq. (6) by a spherical Bessel function, then the plane wave approximation is obtained.

The atomic $T$ matrices and radial matrix elements have been calculated from a self-consistent potential of bulk copper. The latter was obtained with the linear-muffin-tin orbital (LMTO) code $^{20}$ in the local density approximation to density functional theory and the atomic sphere approximation. The dipole matrix elements are evaluated with the acceleration form of the dipole operator. ${ }^{21}$ In all applications below we consider unpolarized light, by adding the intensities for left and right circular polarization.

\section{ANGLE-RESOLVED PHOTOEMISSION SPECTRA}

In this section we focus on energy distribution curves. First, we discuss the DOS and then calculate ARPES. In our realspace method we describe the $\mathrm{Cu}(111)$ surface by a finite semispherical cluster. We have used cluster sizes of 146 and 260 atoms. In the upper panel in Fig. 1, we compare the local DOS at a site in the center of the cluster with the bulk $\mathrm{Cu}$ DOS as obtained with the LMTO band structure method for the infinite crystal.

It can be seen that the RSMS approach succeeds in describing the main features of the bulk DOS, when the calculation is performed using clusters of both 260 and 146 atoms. The main peaks are well reproduced for both cluster sizes. While some discrepancy is observed in the low-energy region, the overall agreement is good. This shows that both clusters are large enough to account for the contribution not

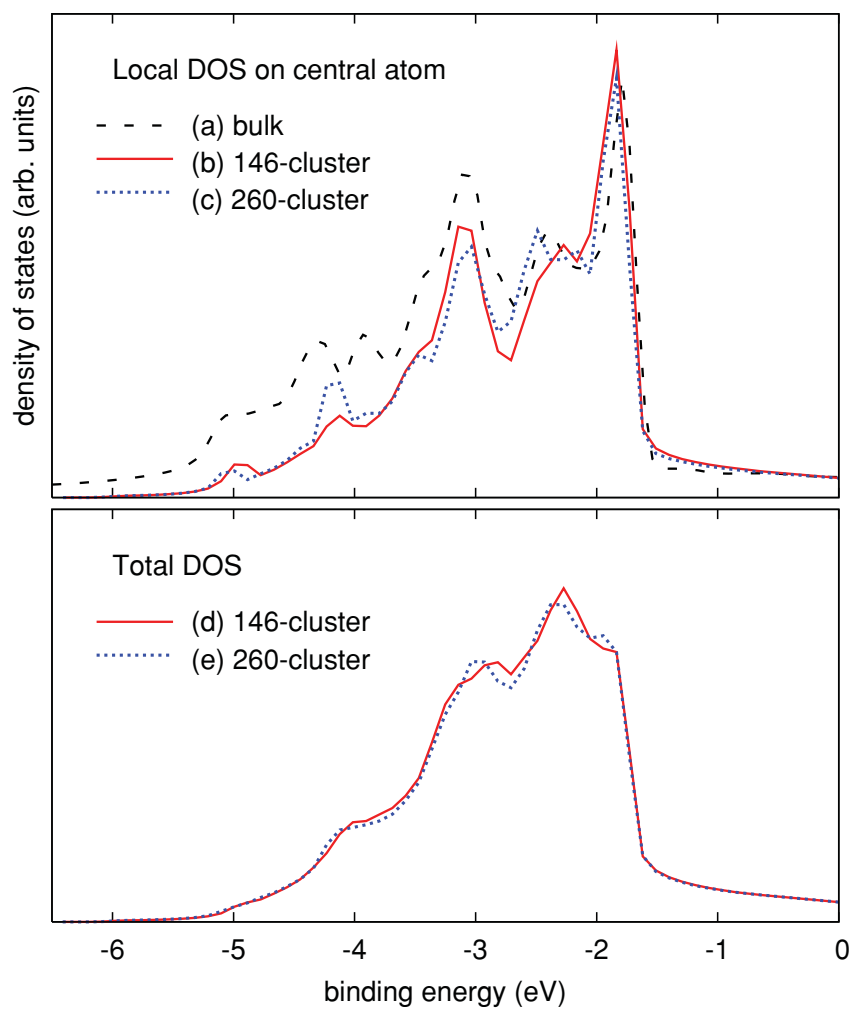

FIG. 1. (Color online) Density of states (DOS). Upper panel: Bulk $\mathrm{Cu}$ DOS from LMTO (a) vs local DOS on a central atom in a 146-atom (b) and a 260-atom (c) $\mathrm{Cu}$ (111) cluster. Lower panel: Total DOS of a 146-atom (d) and a 260-atom (e) cluster. only of the near-surface atoms but also of deeper sites with a bulk-like electronic structure.

The lower panel in Fig. 1 shows the total DOS of the whole cluster, again, for the two cluster sizes. In the total cluster DOS the peaks are smeared out compared to the local DOS of the central atom [Figs. 1(b) and 1(c)]. This difference can be attributed to the undercoordinated surface atoms since the inner atoms have a bulk-like DOS as shown in Figs. 1(b) and 1(c)]. The total cluster DOS hardly changes when going from the 146-atom cluster to the 260-atom cluster; that is, it is well converged for clusters of about 150 atoms.

We now turn to ARPES, that is, we study the variation of the energy distribution curves as a function of the photoelectron emission direction $\left(\mathbf{k}^{\prime}\right)$. ARPES is the main experimental technique for study of band structures. Thus the following results will show to which extent our real-space multiple scattering approach is sensitive to band structure. In Fig. 2 we present results for unpolarized light of $16.8 \mathrm{eV}$ at normal incidence and fixed polar emission angle $\theta=45^{\circ}$. For easy comparison with the experimental data of Ilver and Nilsson, ${ }^{22}$ all calculated spectra have been rescaled in intensity by a constant factor and broadened with a Gaussian of width $0.1 \mathrm{eV}$. Density functional theory systematically underestimates the binding energy of the $\mathrm{Cu}-d$ band states by a few tenths of an electron volt..$^{23,24}$ This has been attributed to self-energy effects which may be taken into account using the GW approximation. ${ }^{25}$ The calculation of the self-energy lies beyond the scope of the present study. Here we correct for its major effect in a phenomenological way by shifting the calculated spectra rigidly by $-0.3 \mathrm{eV}$ in all comparisons with experiment (Figs. 2-6).

Figure 2 shows that the overall agreement between theory and experiment is very good. Almost all peaks are well reproduced in both position and intensity. The evolution of

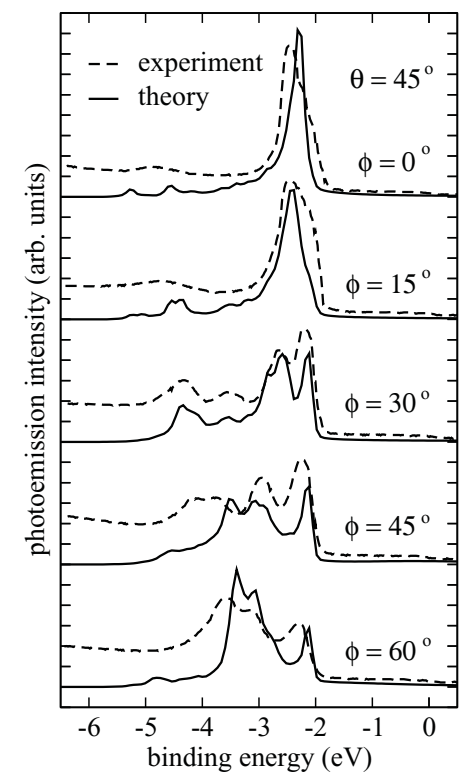

FIG. 2. Photoelectron spectra from $\mathrm{Cu}(111)$ obtained with 16.8-eV light for polar emission angle $\theta=45^{\circ}$ and various azimuthal angles $\phi$ with respect to [211]. Theoretical spectra (solid lines) have been shifted by $-0.3 \mathrm{eV}$ in energy. Relative intensities have not been rescaled. Experimental data (dashed lines) are taken from Ref. 22. 

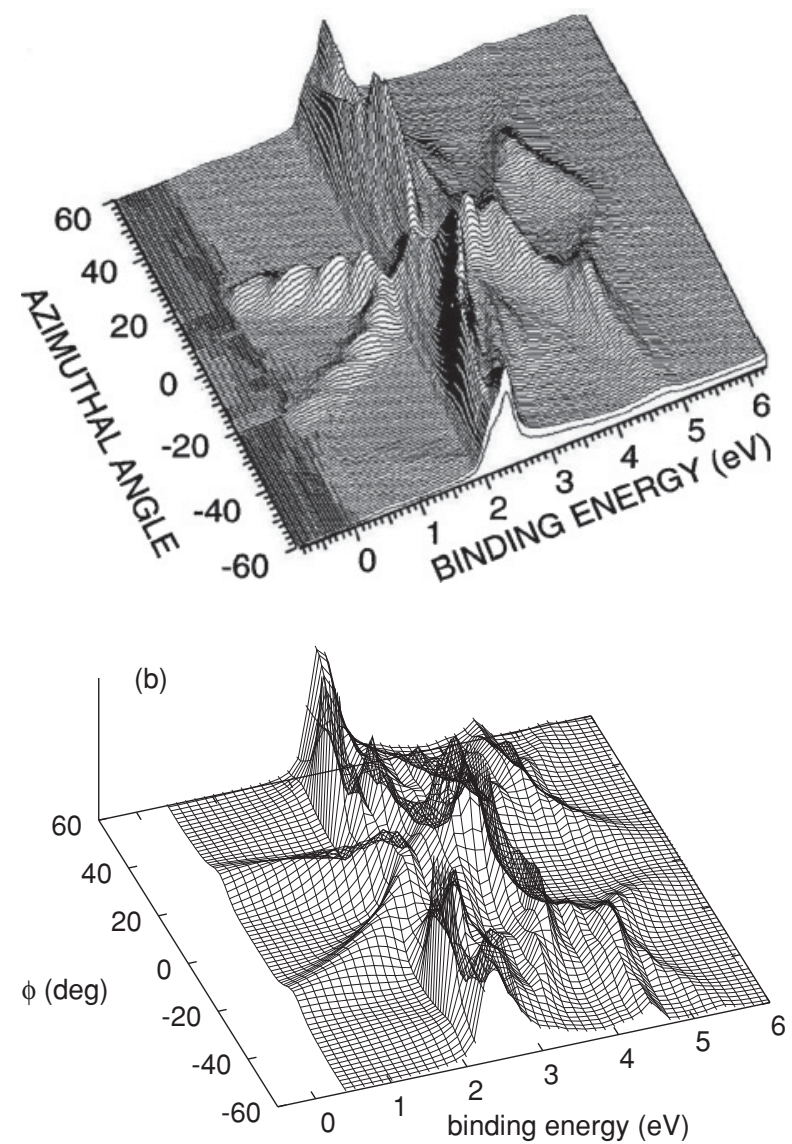

FIG. 3. Photoelectron spectra from $\mathrm{Cu}(111)$ with 21.2-eV light (He I) for $\theta=60^{\circ}$. (a) Experimental data reprinted from Fig. 3 of Ref. 9. Note that the ripples in the parabolic feature at $0-2 \mathrm{eV}$ are an artifact due to the numerical data treatment. (b) Calculated spectra shifted by $0.3 \mathrm{eV}$ to a higher binding energy.

the line shape as a function of azimuthal angle $\phi$ agrees particularly well with the data. The agreement with experiment is as good as the one obtained by Jepsen, ${ }^{23}$ who used a layered KKR scheme.

For another comparison with experiment, we now consider He I light $(21.2 \mathrm{eV})$ and polar angle $\theta=60^{\circ}$. Figure 3(a) shows the experimental ARPES data taken from Ref. 9, and Fig. 3(b), the calculated spectra. Also, in this case, the agreement between theory and experiment is good, not only for the $3 d$ band in the range $2-5 \mathrm{eV}$ but also for the parabolic $s p$-like band, which is seen at binding energies below $2 \mathrm{eV}$.

The results obtained in this section show that the present RSMS approach is suitable for ARPES calculations despite the fact that we do not take advantage of the $k_{\|}$conservation that holds for (perfect) crystal surfaces. The appropriate cluster size will in general depend on the system and the required spectral resolution. In the present case of $\mathrm{Cu}$ ARPES, it is found that a relative small cluster size of about 150 atoms yields sufficiently converged spectra.

\section{PHOTOELECTRON DIFFRACTION PATTERNS}

Here we study PED from the $\mathrm{Cu}-3 d$ band excited with He I (21.2-eV) or He II (40.8-eV) light. The calculated PED patterns are compared with experimental data of
Osterwalder et al. ${ }^{9,16}$ Following the procedure used in these experimental papers, we normalize the PED intensity for each azimuthal scan individually, to remove possible polar angle variations of purely instrumental origin. We thus consider the normalized intensity $I_{n}(\theta, \phi) \equiv I_{b}(\theta, \phi) / \bar{I}_{b}(\theta)$, where $I_{b}(\theta, \phi)$ is the bare intensity and $\bar{I}_{b}(\theta)$ is its average over $0<\phi<360^{\circ}$. The angular mesh is stereographically projected and the intensities $I_{n}(\theta, \phi)$ are plotted in a linear gray scale. Such a plot is called a PED pattern in the following. The calculated patterns in Figs. 4-6 were obtained with the 146-atom cluster. The 260-atom cluster gave almost-identical PED patterns, which are not shown here. In the experiments the photocurrent was recorded for some finite energy window $\left(E_{1}-E_{2}\right)$, that is, the photoemission intensity is integrated over this energy interval. First, we have considered three windows, each $1.2 \mathrm{eV}$ wide, which cover the $\mathrm{Cu}-3 d$ band. The experimental binding energy windows are $\mathrm{W} 1=(2.0-3.2), \mathrm{W} 2=(3.2-4.4)$, and $\mathrm{W} 3=$ (4.4-5.6) eV. For the calculations, these intervals have been rigidly shifted by $-0.3 \mathrm{eV}$ to match the experimental $d$-band position, as before. The experimental ${ }^{16}$ and calculated PED patterns for these energy windows are shown in Fig. 4. In this work all PED patterns were calculated from nonsymmetrized data. The $\mathrm{Cu}(111)$ surface has $C_{3 v}$ symmetry. While the threefold symmetry is fully respected in the PED patterns, the mirror symmetry is only approximate, that is, the patterns
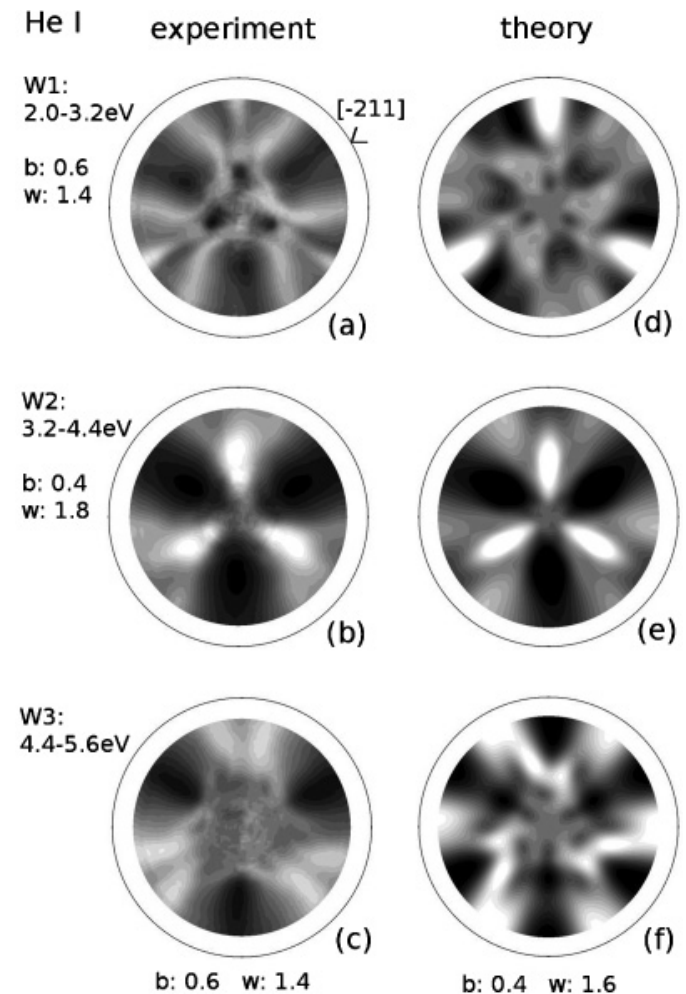

FIG. 4. Experimental $(\mathrm{a}-\mathrm{c})$ and calculated $(\mathrm{d}-\mathrm{f})$ photoelectron diffraction (PED) patterns from $\mathrm{Cu}(111)$ with $\mathrm{He} \mathrm{I}$ light $(21.2 \mathrm{eV})$, for three energy windows in the $\mathrm{Cu}-3 d$ band. The hemisphere is stereographically projected on the disk with the azimuthal orientation fixed by the [-211] direction at 2 o'clock. PED intensities are normalized (see text) and plotted on a linear gray scale with the values for black (b:) and white (w:) as indicated. 
display a $C_{3}$ symmetry. This is because in the experimental setup ${ }^{9}$ the incoming light vector does not lie in a mirror plane.

In Fig. 4, both experimental and theoretical PED patterns vary strongly between the different energy intervals. In principle, some variation can be due to final-state scattering since the kinetic energy of the photoelectrons changes from one energy window to the next by $1.2 \mathrm{eV}$. In particular cases, such as molecules at shape resonances, a change of final-state energy by a few electron volts can indeed lead to strong variations of the PED patterns. In the present system, however, this effect is negligible, as we have checked explicitly by calculating PED patterns, where the initial-state energy window is fixed and the final-state energy window is changed by 1.2 or $2.4 \mathrm{eV}$ (see Appendix A). The same conclusion was reached by Osterwalder et al. ${ }^{9}$ through single scattering calculations with localized initial states. Therefore, the strong variation of the PED pattern as a function of energy seen in Fig. 4 is an almost-pure initial state effect. It is due to the coherent superposition of waves emitted from different sites whose phase relation depends on the initial-state energy. In a molecular bond, for example, the waves emitted from the two atoms are in phase for a bonding state and out of phase by $\pi$ for an antibonding state. Thus the fast variation of the PED patterns across the $\mathrm{Cu}-3 d$ band reflects the delocalized nature of these states. Quite generally, the PED pattern can be expected to vary much faster with initial- than with final-state energy, because the band width, or in other terms the splitting between bonding and antibonding states, is normally much smaller for the valence than the final-state continuum bands.

In Fig. 4, the agreement between experimental and theoretical PED patterns is very good for energy window 2 and fair for windows 1 and 3. Moreover, the evolution of the pattern when going from window 2 to window 3 is qualitatively well reproduced in the calculations, in particular, the shift of the main diffraction peaks to larger polar angles and their splitting into V-shaped features. The calculated patterns display a slightly larger contrast than the experimental ones. This can be expected since in the calculations we have disregarded all broadening effects such as hole decay, inelastic photoelectron scattering, and lattice vibrations.

We have also calculated the PED patterns for He II light $(40.8 \mathrm{eV})$ with the same binding energy windows as for He I (Fig. 4). The He II patterns are shown in Fig. 5. It is seen that also for He II light, the PED pattern changes drastically from

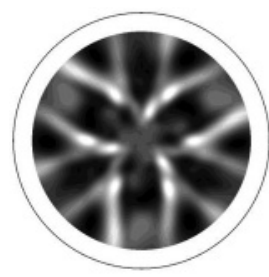

W1: $2.0-3.2 \mathrm{eV}$ b: 0.4 w: 2.0

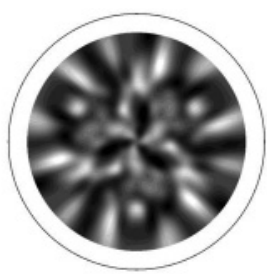

W2: $3.2-4.4 \mathrm{eV}$ b: 0.6 w: 1.6

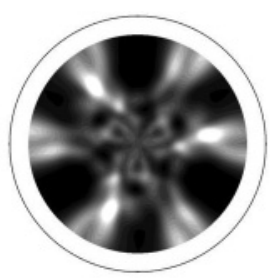

W3: $4.4-5.6 \mathrm{eV}$ b: 0.3 w: 2.4
FIG. 5. Calculated photoelectron diffraction patterns from $\mathrm{Cu}(111)$ with $\mathrm{He}$ II light $(40.8 \mathrm{eV})$, for three energy windows (W1-W3) in the $\mathrm{Cu}-3 d$ band. Binding energy intervals are indicated, as well as black (b:) and white (w:) values on the linear gray scale.
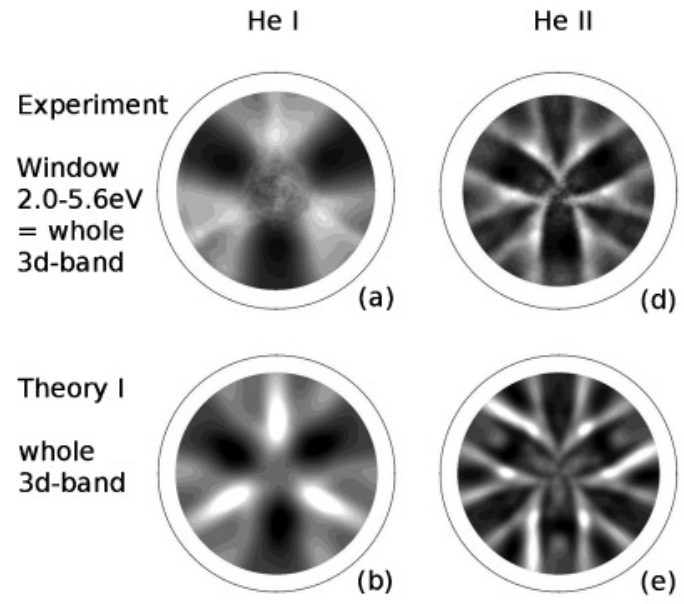

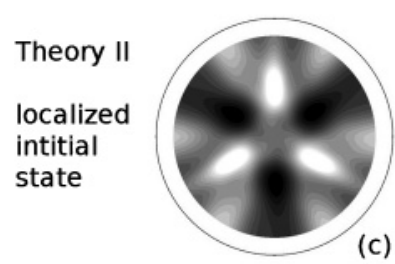

b: 0.6 w: 1.4

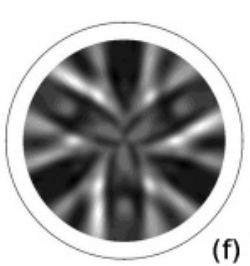

b: 0.6 w: 1.6
FIG. 6. Photoelectron diffraction patterns excited with $\mathrm{He} \mathrm{I} \mathrm{(a-c)}$ and $\mathrm{He}$ II $(\mathrm{d}-\mathrm{f})$ light for an energy window corresponding to the whole $3 d$ band. In Theory II (c, f) localized $\mathrm{Cu}-3 d$ orbitals were used as initial states. The linear gray scale is the same for experimental and theoretical patterns, with normalized intensities for black (b:) and white (w:) as indicated.

one energy window to the next, which is a signature of the delocalized character of the initial $\mathrm{Cu}-3 d$ states.

In Fig. 6, PED patterns for an energy window corresponding to the whole $\mathrm{Cu}-3 d$ band $(2.0-5.6 \mathrm{eV})$ are shown, for excitation with He I and He II light. The experimental data [Figs. 6(a) and 6(d)] is the same as that in Ref. 9. In Fig. 6, "Theory I" [Figs. 6(b) and 6(e)] refers to the same method as in Fig. 4, that is, the full multiple scattering theory for both the initial and the final state. The corresponding PED patterns [Figs. 6(b) and 6(e)] agree very well with experiment [Figs. 6(a) and 6(d)]. While the agreement is excellent for He II light, it is also very good for He I light, except that the theoretical He I patterns have a larger overall contrast. As already mentioned, this is expected because broadening effects were neglected in the calculations. From the comparison between experimental and theoretical patterns in Figs. 4 and 6, we can conclude that the theoretical approach presented in this paper affords an accurate description of valence band PED. It successfully reproduces the observed dependence on both the initial (Fig. 4) and the final state energy (Fig. 6).

The patterns labeled "Theory II" in Figs. 6(c) and 6(f) were calculated using standard XPD theory, that is, from the incoherent sum of photoemission intensities calculated separately for each atom with localized initial states $|v\rangle$ in Eq. (1). For the latter, $\mathrm{Cu}-3 d$ orbitals with an energy in the middle of the $3 d$ band were used. Formally, Theory II is obtained from Theory I by the substitution $\tau_{i j} \rightarrow \delta_{i j} t_{i}$ in Eq. (4). The initial states of Theory II are the same as in Osterwalder et al. ${ }^{9}$ The final states are different, however: single scattering theory 
was used in Ref. 9, but full multiple scattering here. When we compare the PED patterns in Figs. 6(c) and 6(f) with those in Ref. 9, we find only small differences. This is unexpected for the present photoelectron energies below $50 \mathrm{eV}$ where multiple scattering effects are assumed to be large. Recently, Sébilleau and Natoli ${ }^{26}$ investigated this issue and showed that $\mathrm{Cu}-3 d$ emission is a rather particular case, where the multiple scattering series converges for energies as low as $20 \mathrm{eV}$ and so final-state multiple scattering effects are weak.

We now turn to the comparison of the calculated patterns with Theory I versus Theory II in Fig. 6. It is seen that the patterns are almost identical. This means that the rather involved description of delocalized initial states in Theory I can be replaced by a simple XPD-type calculation with localized initial states without loss of accuracy. From the results in Figs. 2-5, it is clear that this simplification is only possible for the PED pattern with an energy window corresponding to the whole $\mathrm{Cu}-3 d$ band. An XPD-type calculation as in Theory II can obviously account neither for the energy dependence of the PED patterns in Figs. 4 and 5 nor for the large energy dispersions shown in Figs. 2 and 3. These energy dependencies are genuine band structure effects due to the quantum mechanical interference between waves emitted from valence orbitals on different sites. Precisely this interference is neglected in the single site approximation of Theory II. The question then arises why this approximation works so well for the energy-integrated PED patterns over the whole $\mathrm{Cu}-d$ band (Fig. 6). Osterwalder et al. ${ }^{9}$ argued that the initial states get effectively localized because the initial wave vector is averaged over large parts of the Brillouin zone due to the finite angular resolution, short escape depth, and phonon effects. While these broadening effects may be important for the experimental patterns, they have been completely neglected in the present calculations. So these effects cannot explain why the calculated PED patterns are almost identical between Theory I and Theory II in Fig. 6. In Appendix B we show that the XPD-type Theory II is a good approximation to the exact delocalized Theory I whenever the photoemission intensity is integrated over a filled, not too wide band of pure $l$ character (such as $\mathrm{Cu}-3 d$ ). The crucial point is that in such a situation, all (delocalized) eigenstates in the energy window can be expanded over localized $l$ orbitals using a single, energy-independent radial wave function. When the whole band is integrated over, the sum over delocalized eigenstates can be replaced by a sum over localized basis states by virtue of a unitarian transformation. We further need to assume that the optical transition matrix elements between localized basis states and photoemission final states vary only slowly with energy such that the matrix elements can be approximately calculated using a single, average final-state energy in the middle of the window. The quality of this second approximation can be appreciated by looking at the rate at which core-level XPD patterns vary as a function of the final-state energy, since the variation comes from the same type of matrix elements. For an energy range of a few electron volts ( $\mathrm{Cu}-d$ band width), the variation of XPD patterns is usually very small, so the approximation is well justified. For the present case of $\mathrm{Cu}(111)$ PED with He I or He II light, the very weak dependence on final-state energy is explicitly shown in Appendix A.
Our proof that delocalized Theory I and XPD Theory II are equivalent for band-integrated photoemission is relevant not only for valence-band, but also for core-level photoemission. Very recently it was shown experimentally that the C- $1 s$ core level in graphene displays a nonzero band dispersion. ${ }^{27}$ This means that even these deep core states are partially delocalized, and so interference between different emitter sites takes place, which is neglected in XPD theory. In a standard photoemission experiment, XPD theory can nevertheless be used without loss of accuracy, because the energy window is much wider than the core-level "band" width of a few tens of milli-electron volts. ${ }^{27}$

\section{CONCLUSIONS}

We have developed a real-space multiple scattering method for valence-band photoemission in the UV regime and have applied it to ARPES and PED of $\mathrm{Cu}(111)$. The numerical results show that the RSMS approach is able to reproduce all essential features of valence band spectra in both angleand energy-resolved mode. The peak dispersion in the ARPES data could be well reproduced, which shows that the method is highly sensitive to the band structure of the system, despite the use of finite clusters where the $k_{\|}$conservation is not exactly fulfilled. PED patterns have been calculated for finite energy windows in the $\mathrm{Cu}-3 d$ band. The patterns depend strongly on the selected binding energy, which reflects the delocalized character of the valence eigenstates where waves emitted from different sites interfere coherently. Good agreement with the available experimental PED patterns was achieved, especially when the energy window corresponds to the full $\mathrm{Cu}-3 d$ band. Since in the latter case, the PED patterns can also be reproduced using a simple XPD-type theory with localized initial states, it has been argued that a localization of the initial $\mathrm{Cu}-3 d$ states takes places in the photoemission process. ${ }^{9}$ From the present results, we find no evidence for such a localization since all the experimental data could be well reproduced using the delocalized description of the $\mathrm{Cu}-3 d$ states. The fact that a simplified XPD-type calculation with localized initial states also reproduces the the $3 d$-band integrated PED patterns is not proof of a localization of the $\mathrm{Cu}-3 d$ states. Instead we have shown, both analytically and numerically, that the exact theory with delocalized initial states reduces to XPD theory when a filled, pure $l$ band is integrated over. We have thus clarified and proved the conjecture of Osterwalder et al. ${ }^{9}$ that XPD theory can be used for an efficient calculation of energy-integrated valence-band PED.

\section{ACKNOWLEDGMENTS}

We would like to thank D. Sébilleau and C. R. Natoli for fruitful discussions. Financial support of the French Agence Nationale de la Recherche (ANR) under Grant RPED (JCJC05_0152), "Resonant PhotoElectron Diffraction," is gratefully acknowledged. Calculations were performed using HPC resources at DSI-CCUB (Université de Bourgogne).

\section{APPENDIX A: VARIATION OF PED PATTERNS WITH INITIAL- AND FINAL-STATE ENERGY}

In Figs. 7 and 8 we show calculated photoelectron diffraction patterns from $\mathrm{Cu}(111)$ for the three energy windows in 
final state kinetic energy

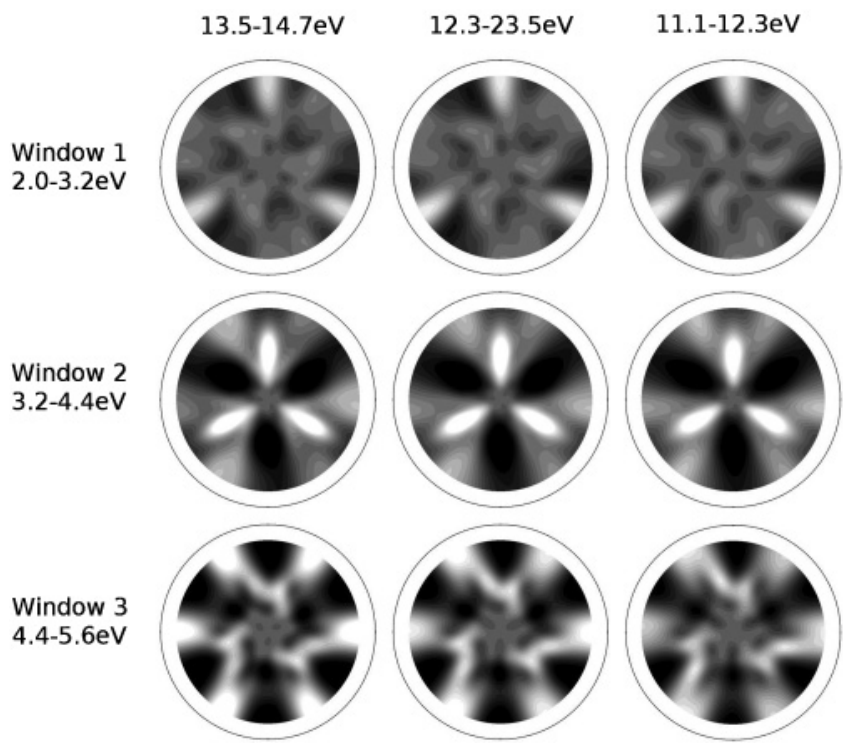

FIG. 7. Calculated photoelectron diffraction patterns from $\mathrm{Cu}(111)$ with a photon energy around $21.2 \mathrm{eV}$ (He I). The same linear gray scale is used for all patterns, with 0.4 for black and 1.8 for white.

the $\mathrm{Cu}-3 d$ band and the final-state energy varied independently in the same range. The patterns on the diagonal in Fig. 7 correspond to He I light, and those on the diagonal in Fig. 8, to He II light. They are identical to the patterns in Figs. 4(d)-4(f) and Fig. 5, respectively (except for slightly different gray scales). The off-diagonal patterns correspond to photon energies shifted by \pm 1.2 or $\pm 2.4 \mathrm{eV}$ such that the initial-state energy window is kept fixed in each row and the

\section{final state kinetic energy}

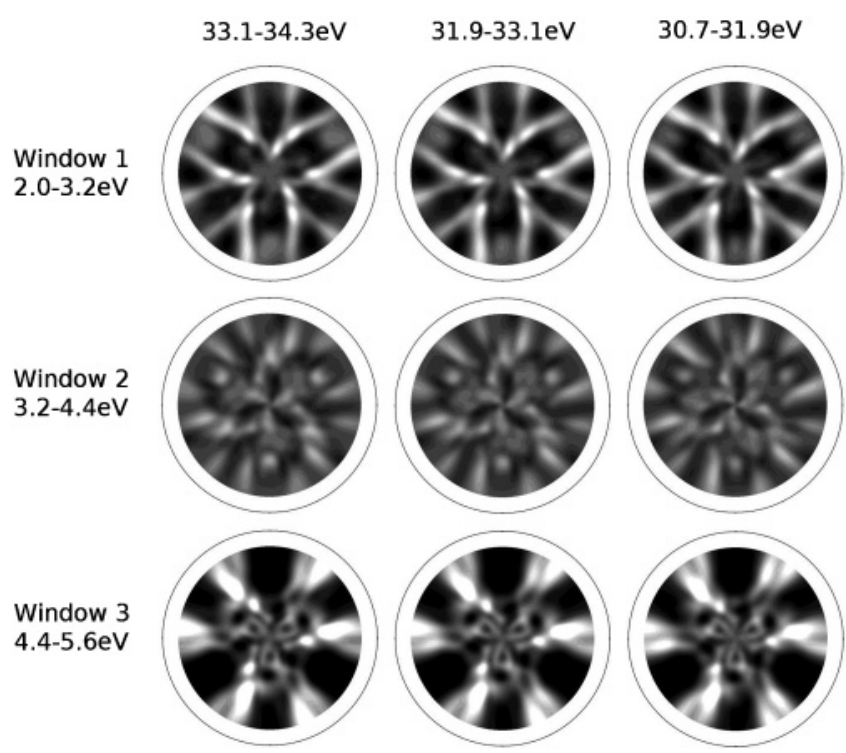

FIG. 8. Calculated photoelectron diffraction patterns from $\mathrm{Cu}(111)$ with a photon energy around $40.8 \mathrm{eV}$ (He II). The same linear gray scale is used for all patterns, with 0.4 for black and 2.0 for white. final-state energy is fixed in each column. The initial binding energy $\left(E_{B}\right)$ and final-state kinetic energy $\left(E_{\text {kin }}\right)$ are indicated. (The photon energy is given by $\hbar \omega=E_{\mathrm{kin}}+W-E_{B}$, with $W=4.5 \mathrm{eV}$.) It can be clearly seen that the PED patterns hardly change when the final-state energy is varied in a range corresponding to the $\mathrm{Cu}-3 d$ width $(3.6 \mathrm{eV})$.

\section{APPENDIX B: XPD APPROXIMATION}

Here we show that the photoelectron intensity integrated over a filled band of pure $l$ character can be approximately calculated using XPD theory, that is, by replacing the exact, delocalized initial states by localized basis orbitals, as we have done in Figs. 6(c) and 6(f). The main assumption is that all eigenstates in the initial energy window can be expanded using a single radial function per site of orbital symmetry $l$ and that the energy window covers the whole set of states spanned by these functions.

Recalling Eq. (1), the photoemission intensity is given by

$$
I\left(\mathbf{k}^{\prime}\right)=\sum_{\nu}\left|\left\langle\mathbf{k}^{\prime}\left|D_{q}\right| v\right\rangle\right|^{2} \delta\left(E_{\mathbf{k}^{\prime}}-E_{v}-\hbar \omega\right) .
$$

We consider an initial energy interval $\left(E_{1}, E_{2}\right)$ that corresponds to a band of pure $l$ character in the tight-binding sense. This means that any eigenstate $|v\rangle$ in this energy interval can be written as a linear combination of localized, energy-independent, and orthonormal basis functions of $l$ symmetry:

$$
|v\rangle=\sum_{i m} C_{i l m}^{v}|i l m\rangle,
$$

where $m$ is the magnetic quantum number. This is a unitarian transformation, that is, we have

$$
\sum_{\nu} C_{i l m}^{\nu} C_{j l m^{\prime}}^{\nu *}=\delta_{i j} \delta_{m m^{\prime}} .
$$

The photoemission intensity becomes

$$
\begin{aligned}
I\left(\mathbf{k}^{\prime}\right)= & \sum_{i j m m^{\prime}}\left\langle\mathbf{k}^{\prime}\left|D_{q}\right| i l m\right\rangle\left\langle j l m^{\prime}\left|D_{q}^{\dagger}\right| \mathbf{k}^{\prime}\right\rangle \\
& \times \sum_{\nu} C_{i l m}^{v} C_{j l m^{\prime}}^{v *} \delta\left(E_{\mathbf{k}^{\prime}}-E_{v}-\hbar \omega\right) .
\end{aligned}
$$

We now consider the photoemission intensity integrated over the interval $\left(E_{1}+\hbar \omega, E_{2}+\hbar \omega\right)$ that is, over the whole $l$ band:

$$
\begin{aligned}
I_{\mathrm{int}}\left(\hat{\mathbf{k}}^{\prime}\right) \equiv & \int_{E_{1}+\hbar \omega}^{E_{2}+\hbar \omega} I\left(\mathbf{k}^{\prime}\right) d E_{k^{\prime}}=\sum_{i j m m^{\prime}} \sum_{v} C_{i l m}^{v} C_{j l m^{\prime}}^{v *} \\
& \times \int_{E_{1}+\hbar \omega}^{E_{2}+\hbar \omega}\left\langle\mathbf{k}^{\prime}\left|D_{q}\right| i l m\right\rangle\left\langle j l m^{\prime}\left|D_{q}^{\dagger}\right| \mathbf{k}^{\prime}\right\rangle \\
& \times \delta\left(E_{\mathbf{k}^{\prime}}-E_{v}-\hbar \omega\right) d E_{\mathbf{k}^{\prime}} .
\end{aligned}
$$

The local matrix elements $\left\langle\mathbf{k}^{\prime}\left|D_{q}\right| i l m\right\rangle$ are of the same type as those appearing in core-level XPD theory. They vary rather slowly with final-state energy $E_{k^{\prime}}$ and can thus be approximated using a single, average final-state 
energy $\bar{E}_{f}=\bar{E}_{v}+\hbar \omega$, where $\bar{E}_{v}$ denotes the center of the valence band:

$$
\left\langle\mathbf{k}^{\prime}\left|D_{q}\right| i l m\right\rangle \approx\left\langle\bar{E}_{f}, \hat{\mathbf{k}}^{\prime}\left|D_{q}\right| i l m\right\rangle .
$$

Then the matrix elements can be taken out of the integral and the integration over the $\delta$ can be performed and yields 1 for all $v$. Together with the orthogonality relation, Eq. (B3), we then get

$$
I_{\mathrm{int}}\left(\hat{\mathbf{k}}^{\prime}\right) \approx \sum_{i m}\left|\left\langle\bar{E}_{f}, \hat{\mathbf{k}}^{\prime}\left|D_{q}\right| i \lim \right\rangle\right|^{2} .
$$

This is the desired result since the right-hand side is the XPD formula. *pkruger@u-bourgogne.fr

${ }^{1}$ S. Hüfner, Photoelectron Spectroscopy (Springer-Verlag, Berlin, 1995).

${ }^{2}$ D. Sébilleau, R. Gunnella, Z.-Y. Wu, S. Di Matteo, and C. R. Natoli,

J. Phys. Condens. Matter 18, R175 (2006).

${ }^{3}$ J. J. Barton, Phys. Rev. Lett. 67, 3106 (1991).

${ }^{4}$ S. Thevuthasan, G. S. Herman, A. P. Kaduwela, R. S. Saiki, Y. J. Kim, W. Niemczura, M. Burger, and C. S. Fadley, Phys. Rev. Lett. 67, 469 (1991).

${ }^{5}$ A. Damascelli, Phys. Scr. T 109, 61 (2004).

${ }^{6}$ T. Okuda, J. Lobo-Checa, W. Auwärter, M. Morscher, M. Hoesch, V. N. Petrov, M. Hengsberger, A. Tamai, A. Dolocan, C. Cirelli, M. Corso, M. Muntwiler, M. Klöckner, M. Roos, J. Osterwalder, and T. Greber, Phys. Rev. B 80, 180404 (2009).

${ }^{7}$ A. Damascelli, Z. Hussain, and Z.-X. Shen, Rev. Mod. Phys. 75, 473 (2003).

${ }^{8}$ This is an idealized picture. In reality, the determination of the full 3D band structure through ARPES is complicated by the fact that the wave vector component perpendicular to the surface is not fully conserved in the photoemission process: it broadens due to the finite final-state extinction length, and it distorts during escape into vacuum.

${ }^{9}$ J. Osterwalder, T. Greber, P. Aebi, R. Fasel, and L. Schlapbach, Phys. Rev. B 53, 10209 (1996).

${ }^{10}$ A. Stuck, J. Osterwalder, T. Greber, L. Schlapbach, R. C. Albers, and M. Alouani, Phys. Rev. B 51, 9497 (1995).

${ }^{11}$ Ch. Sondergaard, Ch. Schultz, M. Schonning, S. Lizzit, A. Baraldi, S. Agergaard, M. B. Nielsen, H. Li, and Ph. Hofmann, Phys. Rev. B 64, 245110 (2001).

${ }^{12}$ J. Li, X. Li, H.-J. Zhai, and L.-S. Wang, Science 299, 864 (2003).

${ }^{13}$ T. Kondow and F. Mafune, Progress in Experimental and Theoretical Studies of Clusters (World Scientific, Singapore, 2003).
${ }^{14}$ N. Berrah, D. Rolles, Z. D. Pesic, M. Hoener, H. Zhang, A. Aguilar, R. C. Bilodeau, E. Red, J. D. Bozek, E. Kukk, R. Diez Muino, and F. J. Garcia de Abajo, Eur. Phys. J. Special Topics 169, 59 (2009).

${ }^{15}$ M. P. Prange, J. J. Rehr, G. Rivas, J. J. Kas, and John W. Lawson, Phys. Rev. B 80, 155110 (2009).

${ }^{16}$ J. Osterwalder, A. Stuck, T. Greber, P. Aebi, L. Schlapbach, S. Hüfner, in Proceedings of the 10th International Conference on Vacuum Ultraviolet Radiation Physics (VUV-10), edited by F. J. Wuilleumier, Y. Petroff, and I. Nenner (World Scientific, Singapore, 1993), p. 475.

${ }^{17}$ In $T$-matrix normalization the $\phi$ 's match to the following free waves at the atomic cell boundary $\phi_{i L}\left(\mathbf{r}, E_{k}\right)=\left[t_{i l}^{-1}\left(E_{k}\right) j_{l}\left(k r_{i}\right)+\right.$ $\left.i k h_{l}^{-}\left(k r_{i}\right)\right] Y_{L}\left(\mathbf{r}_{i}\right)$, where $t, Y, j$, and $h$ denote, respectively, the $T$ matrix, spherical harmonics, Bessel and Hankel functions, and $\mathbf{r}_{i} \equiv \mathbf{r}-\mathbf{R}_{i}$.

${ }^{18}$ K. Wood and J. B. Pendry, Phys. Rev. Lett. 31, 1400 (1973).

${ }^{19}$ J. M. Maclaren, S. Crampin, D. D. Vvedensy, and J. B. Pendry, Phys. Rev. B 40, 12164 (1989).

${ }^{20}$ O. K. Andersen, Phys. Rev. B 12, 3060 (1975).

${ }^{21}$ J. B. Pendry, Surf. Sci. 57, 679 (1976).

${ }^{22}$ L. Ilver and P. O. Nilsson, Solid State Commun. 18, 677 (1976).

${ }^{23}$ D. W. Jepsen, Phys. Rev. B 20, 402 (1979).

${ }^{24}$ V. N. Strocov, R. Claessen, G. Nicolay, S. Hüfner, A. Kimura, A. Harasawa, S. Shin, A. Kakizaki, H. I. Starnberg, P. O. Nilsson, and P. Blaha, Phys. Rev. B 63, 205108 (2001).

${ }^{25}$ A. Marini, G. Onida, and R. Del Sole, Phys. Rev. Lett. 88, 016403 (2001).

${ }^{26}$ D. Sébilleau and C. R. Natoli, J. Phys. Conf. Ser. 190, 012002 (2009).

${ }^{27}$ S. Lizzit, G. Zampieri, L. Petaccia, R. Larciprete, P. Lacovigi, E. D. L. Rienks, G. Bihlmayer, A. Baraldi, and P. Hofmann, Nature Phys. 6, 345 (2010). 